Please do not remove this page

RMIT

UNIVERSITY

\title{
Ergonomic Path Planning for Autonomous Vehicles - An investigation on the effect of transition curves on Handling Behaviour
}

Siddiqi, Muhammad Rehan; Saharkhiz, Alireza; Nakhaie Jazar, Reza; Marzbani, Hormoz

https://researchrepository.rmit.edu.au/esploro/outputs/9921990408301341/filesAndLinks?institution=61RMIT_INST\&index=null

Siddiqi, M. R., Saharkhiz, A., Nakhaie Jazar, R., \& Marzbani, H. (2020). Ergonomic Path Planning for Autonomous Vehicles - An investigation on the effect of transition curves on Handling Behaviour. Proceedings of the 1st International Conference on Information Technology, Advanced Mechanical and Electrical Engineering (ICITAMEE 2020), 50-55. https://doi.org/10.1109/ICITAMEE50454.2020.9398321 Document Version: Accepted Manuscript

Published Version: https://doi.org/10.1109/ICITAMEE50454.2020.9398321

Repository homepage: https://researchrepository.rmit.edu.au (C) 2020 IEEE

Downloaded On 2023/04/26 23:25:09 +1000 


\title{
Ergonomic Path Planning for Autonomous Vehicles - An investigation on the effect of transition curves on Handling Behaviour
}

\author{
Muhammad Rehan Siddiqi*, Alireza Saharkhiz ${ }^{\dagger}$, Reza.N.Jazar ${ }^{\ddagger}$ and Hormoz Marzbani ${ }^{\S}$ \\ School of Mechanical and Automotive Engineering \\ Royal Melbourne Institute of Technology, Melbourne, Australia \\ *Email:s3760026@student.rmit.edu.au, † Email: s3755133@student.rmit.edu.au, \\ ${ }^{\ddagger}$ Email:reza.nakahiejazar@rmit.edu.au and ${ }^{\S}$ Email:hormoz.marzbani@rmit.edu.au
}

\begin{abstract}
As vehicles transcend into higher levels of automation, a key factor that will determine a comfortable driving experience for the passengers will be the handling behaviour of the autonomous vehicles. Path planning will serve an important role in ensuring smooth and comfortable handling. The present study develops a well defined universal scale to define the handling behaviour of autonomous vehicles using different ergonomic path planning tools. Firstly, a reference track is designed to incorporate the worst handling. Followed by designing ergonomic roads using transition curves such as B-spline, Bezier and Hermite curves to investigate the impact of these curves on handling behaviour. Trajectory tracking of an 8 degree of freedom vehicle model is then studied using curvature dependent and curvature independent controllers to draw a comparison. Results are then compared and evaluated using a universal cost function to determine the optimal transition curve for handling. Results indicate that 3-point defined curves in general perform better handling with $B$-spline evaluated as the best handling transition curve for 4-point defined curves. Further research is recommended by utilising key characteristics of the transition curves like local control and non-uniformity.
\end{abstract}

\section{INTRODUCTION}

$\mathbf{S}$ ELF-DRIVING cars have the potential to lead to significant benefits. From the driver's perspective, the direct benefits of this technology are considered to be increased comfort and productivity. At the time of writing this document some levels of automation have already been introduced to the vehicles such as forward collision warning, to adaptive cruise control and lane keeping to fully automated driving. According to the Society of Automotive Engineers [1] there are 5 levels of automation. From level 0 which is the absence of automation to level 4, full automation. Introduction of such systems has raised several classic human factor issues [2]. Of particular immediate concern are the questions that arise at automation level 3. At this level which is known as "Conditional Automation" the driver is expected to resume vehicle control with a sufficiently comfortable transition time in case the system reaches its performance limits, or because the driver desires to return to manual drive. The safe and comfortable transitioning between in-the-loop and out-of-theloop behaviours raises several questions [2]. In a more general sense the fact that the passengers of such vehicles will face loss of control has also raised more human factor questions [3](loss of control-ability [4]). Passenger comfort in ground transportation is determined by the changes in the motion felt in all directions [4]. Many studies though have focused on the comfort levels in the longitudinal direction. Such studies are limited to acceleration or jerk limits in longitudinal motion and study its effect on passenger comfort while ignoring lateral motion [5]. Authors in [6] also tried to introduce a definition for human-comfort in autonomous driving for passenger vehicle navigation. An electric wheelchair was used as the platform and the objective result was a path with a better view of the other sides of turns to be more comfortable. Human comfort in terms of enhancing the controller was discussed and optimised in [7]. A cost function was developed based on five factors defined using techniques like root mean square (RMS) and fast Fourier transforms (FFT), the cost function was then used to optimise the controller to follow the paths defined using Clothoids in the most comfortable manner. However, the effect of handling behaviour of an autonomous vehicle due to transition curves has never been evaluated on a universal scale with the use of sophisticated controllers. The present study investigates the handling behaviour of an autonomous vehicle in terms of comfort by deploying different transition curves on a reference track. Various scenarios are then simulated using the Autodriver algorithm which benefited from the use of Sliding Mode Control (SMC) as well as Model Predictive Controller (MPC) as trajectory tracking mechanisms. Lastly a well defined cost function is used to evaluate different scenarios generated on a universal scale.

\section{PATH-TRACKING CONTROL}

\section{A. Autodriver algorithm}

Employing the concept of center and radius of curvature of the road, and the dynamic rotation center of the vehicle, Jazar [8] introduced a method for calculating the required steer angle to keep the vehicle on the given road. This is done by coinciding the center of curvature of the vehicle and the road. The algorithm was later further developed to use simpler methods for calculation of steering angles where close-to-dynamically-precise steering angles could be produced using simple kinematic equations [9]. The algorithm was farther equipped with control mechanisms to assure a practical approach [10]. 


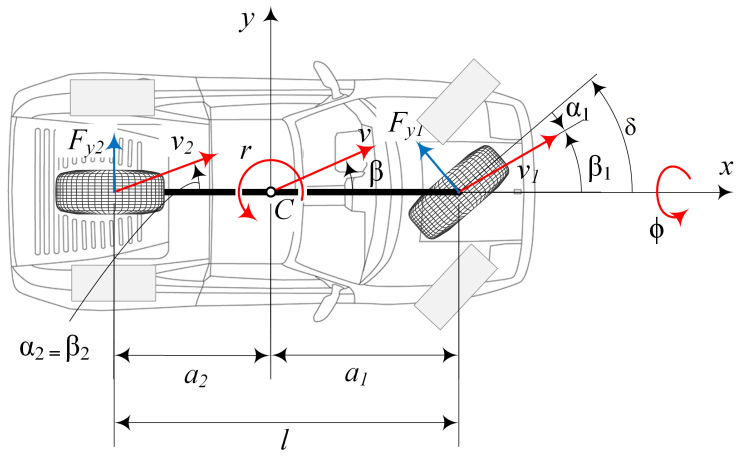

Fig. 1. Bicycle-Roll Vehicle Model

To use the Autodriver algorithm as a trajectory tracking mechanism, a desired path of motion given as a mathematical equation is needed. This feature makes the algorithm a suitable platform for the current study.

1) Vehicle modeling for Autodriver algorithm: The bicycleroll model of a vehicle utilized in this study is shown in Figure 1. The model is based on the nonlinear coupled ordinary differential equations (Equations 1 - 19 ) governing the motion of a rigid roll-able vehicle moving in the osculating plane of the road.

$$
\begin{gathered}
\dot{v_{x}}=F_{x} / m+r v_{y} \\
\dot{v_{y}}=\left(\frac{C_{r}}{m}-v_{x}\right)+\frac{C_{p}}{m} p+\frac{C_{\beta}}{m} \frac{v_{y}}{v_{x}}+\frac{C_{\alpha}}{m} \alpha+\frac{C_{\delta}}{m} \delta \\
\dot{p}=\frac{1}{I_{x}}\left(E_{r} r+E_{p} p+E_{\beta} \frac{v_{y}}{v_{x}}+E_{\alpha} \alpha+E_{\delta} \delta\right) \\
\dot{r}=\frac{1}{I_{z}}\left(D_{r} r+D_{p} p+D_{\beta} \frac{v_{y}}{v_{x}}+D_{\alpha} \alpha+D_{\delta} \delta\right)
\end{gathered}
$$

where,

$$
\begin{gathered}
C_{r}=\frac{\partial F_{y}}{\partial r}=-\frac{a_{1}}{v_{x}} C_{\alpha f}+\frac{a_{2}}{v_{x}} C_{\alpha r} \\
C_{p}=\frac{\partial F_{y}}{\partial p}=\frac{C_{\alpha f} C_{\beta f}}{v_{x}}+\frac{C_{\alpha r} C_{\beta r}}{v_{x}} \\
C_{\beta}=\frac{\partial F_{y}}{\partial \beta}=-\left(C_{\alpha f}+C_{\beta f}\right) \\
C_{\phi}=\frac{\partial F_{y}}{\partial \phi}=C_{\alpha r} C_{\delta \phi r}+C_{\alpha f} C_{\delta \phi f}-C_{\phi f}-C_{\phi r} \\
C_{\delta}=\frac{\partial F_{y}}{\partial \delta}=C_{\alpha f} \\
D_{r}=\frac{\partial M_{z}}{\partial r}=-\frac{\left(a_{1}\right)^{2}}{v_{x}} C_{\alpha f}-\frac{\left(a_{2}\right)^{2}}{v_{x}} C_{\alpha r} \\
D_{p}=\frac{\partial M_{z}}{\partial p}=\frac{a_{1}}{v_{x}} C_{\beta f} C_{\alpha f}-\frac{a_{2}}{v_{x}} C_{\beta r} C_{\alpha r}
\end{gathered}
$$

$$
\begin{gathered}
D_{\beta}=\frac{\partial M_{z}}{\partial \beta}=-\left(a_{1}\right) C_{\alpha f}-\left(a_{2}\right) C_{\alpha r} \\
D_{\phi}=\frac{\partial M_{z}}{\partial \phi}=-\left(a_{1}\right)\left(C_{\alpha f}-C_{\alpha f} C_{\delta \phi f}\right) \\
+\left(a_{2}\right)\left(C_{\phi r}-C_{\alpha r} C_{\delta \phi r}\right) \\
D_{\delta}=\frac{\partial M_{z}}{\partial \delta}=a_{1} C_{\alpha f} \\
E_{r}=\frac{\partial M_{z}}{\partial r}=-\frac{a_{1}}{v_{x}} C_{T f} C_{\alpha f}+\frac{a_{2}}{v_{x}} C_{T r} C_{\alpha r}
\end{gathered}
$$

$$
\begin{gathered}
E_{r}=\frac{\partial M_{z}}{\partial p}=\frac{1}{v_{z}} C_{\beta f} C_{T f} C_{\alpha f}+\frac{1}{v_{z}} C_{\beta r} C_{T r} C_{\alpha r}-C_{\alpha} \\
E_{\beta}=\frac{\partial M_{z}}{\partial \beta}=-C_{T f} C_{\alpha f}-C_{T r} C_{\alpha r} \\
E_{\alpha}=\frac{\partial M_{z}}{\partial \alpha}=-C_{T f}\left(C_{\alpha f}-C_{\alpha f} C_{\delta \phi f}-k_{\phi}\right. \\
-C_{T r}\left(C_{\alpha r}-C_{\alpha r} C_{\delta \phi r}\right) \\
E_{\beta}=\frac{\partial M_{z}}{\partial \delta}=-C_{T f} C_{\alpha f}
\end{gathered}
$$

The input to the system is steering angle $\delta$. The forward velocity is assumed to be a varying parameter for a third order system constructed by 2-4. The tire forces are assumed to be proportional to the side-slip angles. Also, a single track layout is assumed which considers the effect of the left and right tires by using a single equivalent tire in the middle of the axle.

For the sake of real time application of Autodriver algorithm a technique called steady state dynamic steering was introduced which is based on the use of the steady state responses of the vehicle [9] [11]. Autodriver algorithm uses the fact that a vehicle on the road must always rotate about the center of curvature of that road to coincide it with the center of rotation of the vehicle. Knowing the mathematical equation of any road as a spatial curve $(\vec{r}=\vec{r}(X, Y, Z, \psi))$ enables the calculation of the path of curvature center in the osculation plane, in global and vehicle body coordinate frames. Assuming there is no initial position error between the vehicle and the road, if the location of the road curvature center coincides with the vehicle's rotation center at every instance, the vehicle would remain on the road. To compensate for transient errors and any uncertainties in modeling, corrective input steering $\delta$, and $v_{x}$ are selected.

The turning radius of the vehicle is mainly determined by the steer angle. Although the velocity does have some contribution to the Instantaneous Center of Rotation (ICR) location, it is less effective in determining the turning radius, especially at small side-slip angles [12]. Hence, the main factor determining the radius of rotation of the vehicle is assumed to be the steer angle. Thus, the aim is to set the steer angle to eliminate the position error between the ICR and the road curvature center laterally; then the velocity of the vehicle will impose a certain side-slip angle $\beta$ to the vehicle, which eventually, matches the longitudinal position of ICR in body 
coordinate with the road curvature center [12]. The desired velocity is normally given by the high-level path planner as a velocity profile for different sections of a road.

\section{B. Control strategy}

The main portion of the required steering action in transient maneuvering is constituted by steer angle calculated by the Autodriver algorithm. To completely eliminate transient tracking errors, a corrective steering signal is required. Thus, Autodriver is used as feedforward and a closed-loop controller is added as feedback. The longitudinal control is performed using a separate closed-loop Proportional-Derivative (PD) controller. Due to the inherent double integration involved in achieving the longitudinal position from longitudinal acceleration (as the manipulated variable), usage of integral control is avoided to eliminate integral windup. The steady-state error is negligible in the longitudinal direction compared to the lateral one, which is the main control objective.

For the purpose of simulation, actual posture of the vehicle in the global frame is calculated as:

$$
\begin{gathered}
X=X_{0}+\int_{0}^{t}\left(v_{x} \cos \psi-v_{y} \sin \psi\right) d t \\
Y=Y_{0}+\int_{0}^{t}\left(v_{x} \sin \psi+v_{y} \cos \psi\right) d t \\
\psi=\psi_{0}+\int_{0}^{t} r d t
\end{gathered}
$$

The desired vehicle location and its center of curvature are functions of time, which are calculated based on:

$$
\begin{gathered}
{ }^{G} \mathbf{r}_{d}={ }^{G} \mathbf{r}_{d}\left(X_{d}(t), Y_{d}(t), Z_{d}(t)\right) \\
{ }^{G} \mathbf{r}_{c}={ }^{G} \mathbf{r}_{c}\left(X_{c}(t), Y_{c}(t), Z_{c}(t)\right)
\end{gathered}
$$

Two-dimensional roads are studied, hence, the $Z$ coordinate will be zero. This serves as a reference point which ideally follows the desired path with the desired velocity profile at every instance. The goal of the path-tracker is to make the ego vehicle follow the ghost-car on the road.

The overall algorithm may be summarized into the following steps:

- A desired path of motion (23)-(24) given as a functions of time;

- Kinematic transformation provides the coordinates of the road and its curvature center in the vehicle body coordinate frame;

- Steady-state equations determine the feed-forward steer angle $\delta_{s s}=\delta_{f f}$ at the given longitudinal speed $v_{x}$ and the radius of rotation $\rho$;

- The transient lateral error in body coordinate frame, which is equal to the desired vehicle position in the body frame, $e_{y}=y_{d}-y=y_{d}$ is compensated with a feedback signal $\delta_{f b}$ to adjust the total steer angle $\delta=\delta_{f f}+\delta_{f b}$;

- The longitudinal error $e_{x}=x_{d}-x=x_{d}$ is eliminated by a PD controller.
Quick and reliable response of the control system based on the available road data will be ensured using the Autodriver algorithm as feed-forward, even in adverse weather conditions. The transient error, however, needs to be fully compensated in order to achieve a robust autonomous path-tracking performance.

Three controllers have been used to enable the investigation of the effect of control system on the handling and comfort level of the car. The results are extensively discussed in the discussion section. The architecture of the control methods are discussed below.

1) Longitudinal closed-loop control: The longitudinal control is performed using a PD controller that eliminates the longitudinal position errors. The control input to the system in this direction is the longitudinal force $F_{x}$ as in (1). Integral control is not considered here, because the controlled variable has a dimension of length (position), which is inherently the integration of the velocity as the manipulated variable. The calculated longitudinal force required, is converted to a torque input to the driving wheels with the simplified relationship:

$$
T=F_{x} R_{w}
$$

2) Closed-loop steering using Sliding-Mode Control: As the first method, a Sliding-Mode Controller (SMC) is designed to ensure accurate lateral tracking with robust performance with respect to uncertainties [13], specially on tire parameters. The SMC is designed based on a simplified bicycle model [14] with addition of lateral position dynamics as shown in figure 2:

$$
\frac{d}{d t}\left[\begin{array}{c}
v_{y} \\
r \\
\dot{e} \\
e
\end{array}\right]=\left[\begin{array}{cccc}
A_{11} & A_{12} & 0 & 0 \\
A_{21} & A_{22} & 0 & 0 \\
-A_{11} & -A_{12}-v_{x} & 0 & 0 \\
0 & 0 & 1 & 0
\end{array}\right]\left[\begin{array}{c}
v_{y} \\
r \\
\dot{e} \\
e
\end{array}\right]+\left[\begin{array}{c}
B_{1} \\
B_{2} \\
-B_{1} \\
0
\end{array}\right] \delta
$$

where,

$$
\begin{gathered}
A=\left[\begin{array}{cc}
-\frac{C_{\alpha f}+C_{\alpha r}}{m v_{x}} & \frac{-a_{1} C_{\alpha f}+a_{2} C_{\alpha r}}{m v_{x}}-v_{x} \\
-\frac{a_{1} C_{\alpha f}-a_{2} C_{\alpha r}}{I_{z} v_{x}} & -\frac{a_{1}{ }^{2} C_{\alpha f}-a_{2}{ }^{2} C_{\alpha r}}{I_{z} v_{x}}
\end{array}\right] \\
B=\left[\begin{array}{c}
-\frac{C_{\alpha f}}{m} \\
\frac{a_{1} C_{\alpha f}}{I_{z}}
\end{array}\right]
\end{gathered}
$$

The error dynamics $(\ddot{e})$ becomes:

$$
\begin{gathered}
\dot{e}=-v_{y^{\prime}}=-\left(v_{y} \cos \Delta \psi+v_{x} \sin \Delta \psi\right) \approx-v_{y} \Delta \psi v_{x} \\
\ddot{e} \approx-\dot{v}_{y}-r v_{x}
\end{gathered}
$$

Assuming a constant $v_{x}$ and $\frac{d}{d t} \Delta \psi \approx r$, a sliding surface is constructed as:

$$
s=\dot{e}-\lambda e
$$

$$
\dot{s}=\ddot{e}-\lambda e=\dot{v}_{y}+r v_{x}+\lambda e=0
$$




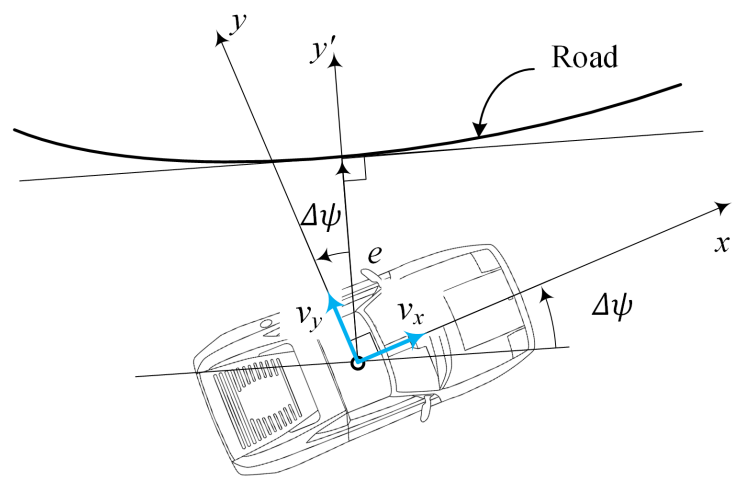

Fig. 2. Path-Following Error

Substituting from (26) into (32), an equivalent control input is calculated which keeps the state trajectories on the sliding surface:

$$
\begin{aligned}
& A_{11} v_{y}+\left(A_{1} 2+v_{x}\right) r+B_{1} \delta_{e q}+\lambda \dot{e}=0 \\
& \delta_{e q}=-\frac{1}{B_{1}}\left[A_{11} v_{y}+\left(A_{12}+v_{x}\right) r+\lambda \dot{e}\right]
\end{aligned}
$$

Adding a regulatory term to (34) compensates deviations due to uncertainties and dynamic simplifications involved in the control design. A saturation function is used for this purpose to reduce chattering in the control signal:

$$
\begin{gathered}
\delta_{f b}=\delta_{e q}-k \cdot \operatorname{sat}\left(\frac{s}{\Phi}\right) \\
\operatorname{sat}(u)=f(x)= \begin{cases}u, & |u| \leq 1 . \\
\operatorname{sgn}(u), & |u|>1 .\end{cases}
\end{gathered}
$$

Controller parameters $\lambda, k, \Phi$ were initially tuned based on Figure 1 and lane-change scenarios using clothoids as transition curves and they have been selected such that the controller satisfies the sliding condition for tire parameter uncertainties of up to 10 percent; the corresponding controller is referred to as "SMC" in this text. Another tuning was later performed for a more aggressive controller based on a wider set of transition curves; the corresponding controller is named and referred to as "SMC-Modified".

3) Closed-loop steering using Model-Predictive Control: To compare the effect of transition curves on control performance and passenger comfort, a Model-Predictive Controller (MPC) is built using (26) as the internal model. The implementation is performed in MATLAB Simulink using "MPsee" toolbox [15] which utilizes Generalized Minimum Residual Method (GMRES) as the optimization tool. A prediction horizon of 10 with a sampling time of $0.1 s$ is considered for the MPC. A summation with counter $\mathrm{k}$ from 1 to 10 is needed over $e_{k}$ and $\delta_{k}$. The cost function is considered as:

$$
J=w_{1} e_{k}^{2}+w_{2} \delta_{k}^{2}
$$

and a constraint is applied to limit the steer angle:
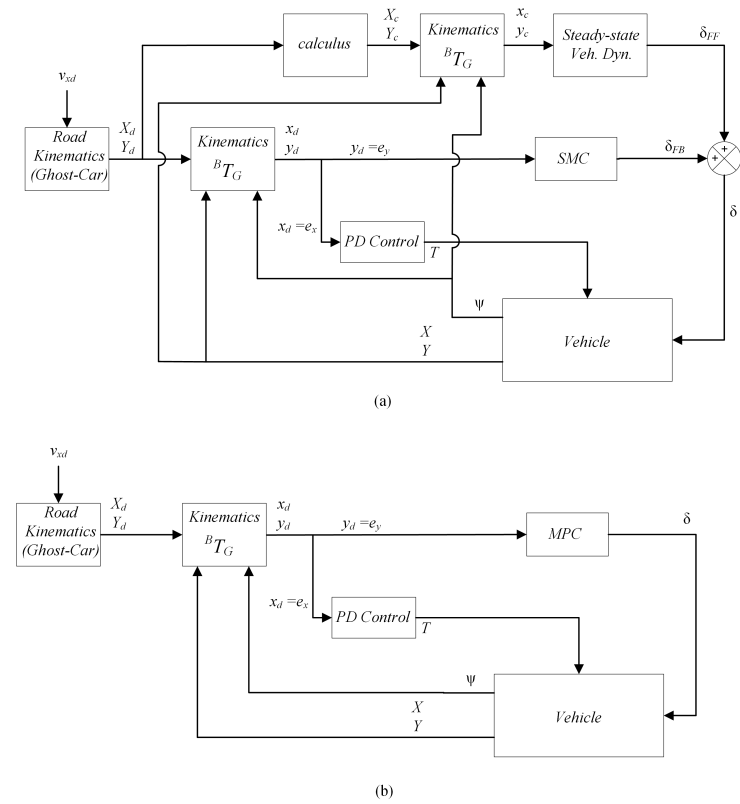

Fig. 3. Block-diagram of the control system: (a) SMC, (b) MPC

TABLE I

CONTROLler PARAMETERS

\begin{tabular}{|c|c|}
\hline Controller & Parameters \\
\hline SMC & $\lambda=1, k=0.15, \Phi=0.15$ \\
SMC-Modified & $\lambda=1, k=0.3, \Phi=5$ \\
MPC & $w_{1}=1, w_{2}=8, \delta_{m}=\pi / 6$ \\
\hline
\end{tabular}

$$
-\delta_{m} \leq \delta \leq \delta_{m}
$$

Block-diagram of the full control system is shown in Figure 3 and the controller parameters are summarized in Table I.

\section{Simulations}

\section{A. Vehicle and tire model}

For the purpose of simulations, a more realistic nonlinear 8 degree of freedom vehicle model is constructed. The model takes into account the longitudinal, lateral, roll, and yaw motions as well as rotations of all four wheels. A combinedslip tire force model called "elliptic tire model" [16] is used to calculate tire forces based on the longitudinal and the side-slip under each tire.

The parameters used in this study are summarized in Table II. It is noteworthy that the parameters of the four-wheel model including the elliptic tire are matched to the bicycle and bicycle-roll models described in this document.

\section{B. Cost Function}

Vehicle comfort has been evaluated in terms of handling behaviour via a cost function first introduced in [7], where the cost function is a combination of Low-frequency Highmagnitude Horizontal, Yaw and Roll motions. The objective functions have been summarised below: 
TABLE II

VEHICLE PARAMETERS

\begin{tabular}{|c|c|c|c|}
\hline Parameter & Value [Unit] & Parameter & Value [Unit] \\
\hline$m$ & $845.5[\mathrm{~kg}]$ & $C_{\delta_{\phi f}}$ & 0.01 \\
$I_{x}$ & $350\left[\mathrm{~kg} \cdot \mathrm{m}^{2}\right]$ & $C_{\delta_{\phi r}}$ & 0.01 \\
$I_{z}$ & $1490\left[\mathrm{~kg} \cdot \mathrm{m}^{2}\right]$ & $C_{\phi_{f}}$ & $-3200[\mathrm{~N} / \mathrm{rad}]$ \\
$C_{\alpha_{f}}$ & $52000[\mathrm{~N} / \mathrm{rad}]$ & $C_{\phi_{r}}$ & $0[\mathrm{~N} / \mathrm{rad}]$ \\
$C_{\alpha_{r}}$ & $64000[\mathrm{~N} / \mathrm{rad}]$ & $R_{w_{i}}$ & $0.35[\mathrm{~m}]$ \\
$C_{T_{f}}$ & $0.4[\mathrm{~m}]$ & $c_{\phi}$ & $1700[\mathrm{Nm} . \mathrm{s} / \mathrm{rad}]$ \\
$C_{T_{r}}$ & $0.4[\mathrm{~m}]$ & $k_{\phi}$ & $26612[\mathrm{Nm} / \mathrm{rad}]$ \\
$C_{\beta_{f}}$ & $-0.4[\mathrm{~s}]$ & $a_{1}$ & $0.909[\mathrm{~m}]$ \\
$C_{\beta_{r}}$ & $-0.1[\mathrm{~s}]$ & $a_{2}$ & $1.436[\mathrm{~m}]$ \\
\hline
\end{tabular}

- RMS Value of the FFT Spectrum of Steering Angle Variation by minimizing High Magnitude Low Frequency values of steering angle aiming not to coincide with human body's resonance frequencies.

$$
O B J_{1}=\sqrt{\operatorname{Mean}\left(F F T_{\delta}^{2}\right)}
$$

- Sum of Vibration Dose Value (VDV) of both Lateral and Roll Accelerations minimizing the effect of Lateral and Roll accelerations on handling behavior (40).

$$
O B J_{2}=V D V_{\text {lateral }}+V D V_{\text {roll }}
$$

- RMS Value of the Lateral Path Tracking Error laterally minimizing the deviation of the predefined AV's trajectory from the main comfortable path profile(41).

$$
O B J_{3}=\sqrt{\text { Mean }\left(\left(Y_{\text {trajectory }}-Y_{\text {path }}\right)^{2}\right)}
$$

The three objective functions are then given appropriate weightages (based on trial and error) and summed to obtain a single scalar representation of the paths handling behaviour as indicated by the equation (42), where a small handling behaviour value indicates high levels of comfort.

$$
\text { Handling }=W_{0} * O B J_{1}+W_{1} * O B J_{2}+W_{2} * O B J_{3}
$$

In order to avail the cost function a reference track was introduced and routed with different transition curves the following sections illustrate these details.

\section{Reference Track}

Previous studies such as [17] and [18] encouraged us to construct the reference track and by designing the reference track using circular arcs we maximised discomfort in handling behaviour, hence labelled as the "Worst path" in our study. The equations utilised are as follows:

$$
\begin{gathered}
x=R \cos (t) \\
y=R \sin (t) \\
R^{2}=(x-h)^{2}+(y-k)^{2}
\end{gathered}
$$

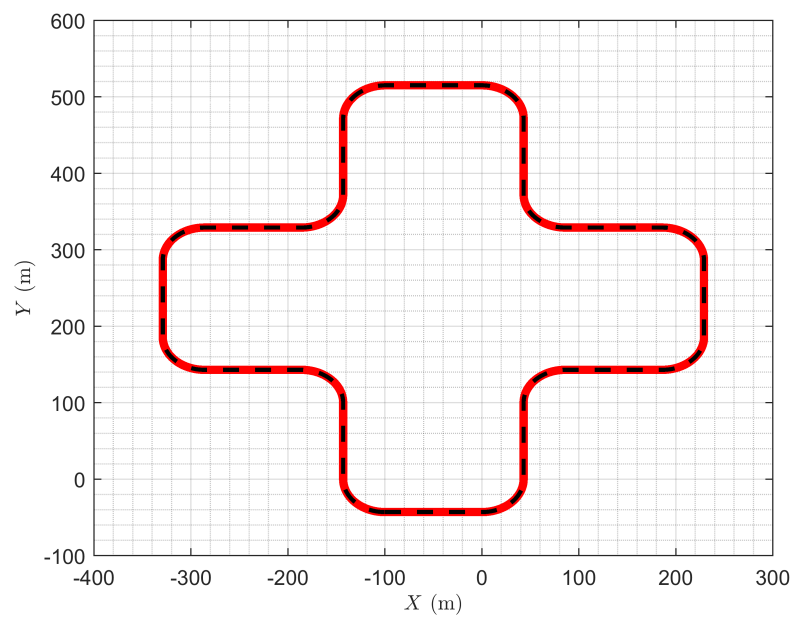

Fig. 4. Reference track generated using equations [43][44][45]

The minimum radius $(\mathrm{R})$ is $43 \mathrm{~m}$, calculated based on a passenger car (Chevrolet Corvette) travelling at $60 \mathrm{~km} / \mathrm{h}(\mathrm{V})$ with a base(b)-to-height(h) ratio of 6.5 , using the overturning ratio below[19]:

$$
\frac{V^{2}}{g R} \leq \frac{(b / 2)}{h}
$$

Using Equations (43)(44)(45) and (46) the Reference track was originated as illustrated in Figure 4.

By applying the cost function developed in the previous section the worst path generated a numerical value, which is illustrated in table III:

TABLE III

HANDLING BEHAVIOUR OF ONLY THE WORST PATH USING THE COST FUNCTION

\begin{tabular}{|c|c|c|c|}
\hline $\mathrm{W}_{0} * O b j_{1}$ & $\mathrm{w}_{1} * O b j_{2}$ & $\mathrm{w}_{2} * O b j_{3}$ & Total \\
\hline 0.66 & 28.8 & 0.44 & 29.3 \\
\hline
\end{tabular}

The value calculated as per our cost function for the "worst path" gives a numerical value for the worst handling behaviour. Using transition curves (described in the following section) we hope to redesign the same worst path and reduce(improve) the numerical value of the handling behaviour.

\section{Transition Curves}

Parametric curves which are currently the most used path planning tools available as suggested by a number of previous studies [18], [20], [21], [22], [23], [24] and [25] are used in the present study.

- Parametric Cubic (Hermite) Curves are defined by four blending functions shown below:

$$
\begin{gathered}
F 1=2 u^{3}-3 u^{2}+1 \\
F 2=-2 u^{3}+3 u^{2}
\end{gathered}
$$




$$
\begin{gathered}
F 3=u^{3}-2 u^{2}+u \\
F 4=u^{3}-u^{2}
\end{gathered}
$$

The Blending functions (47)(48)(49)(50) are defined using a parameter $u$ which ranges from 0 to 1 over the whole curve. where $\mathrm{P}(0)$ and $\mathrm{P}(1)$ are the starting and ending points of the curves respectively. The complete parametric curve equation is given as follows:

$$
C(u)=P(0) F 1+P(1) F 2+\dot{P}(0) F 3+\dot{P}(1) F 4
$$

- Bezier Curves are defined using the nth order based on the below equation:

$$
C(u)=\sum_{i=0}^{n} B_{n, i}(u) P_{i}
$$

The blending functions are defined using the Binomial expansion given by the equation(53):

$$
B_{n, i}(u)=\frac{n !}{i !(n-i) !} u^{i}(1-u)_{n-i}
$$

The advantage of using Bezier curves is that arbitrary points can be selected to define the path, but at the cost of increasing the order of the curve, making the equation of the curve more complex whilst having only global control over the track/path.

- B-Spline Curves define a large Curve into smaller segments by using a knot vector $t_{i}$ and the blending functions are defined using the Basis function defined in equation(54):

$$
\begin{aligned}
N_{i, k}(u) & =\frac{\left[u-t_{i}\right]}{\left[t_{i+k-1}-t_{i}\right]} N_{i, k-1}(u) \\
& +\frac{\left[t_{i+k}-u\right]}{t_{i+k}-t_{i+1}} N_{i+1, k-1}(u)
\end{aligned}
$$

In order to simplify the calculation of the Basis function Cox-de Boor algorithms are used[26], which are either equal to a 1 or 0 depending upon their existence in the concerned segment, as shown below:

$$
N_{i, 0} \begin{cases}=1 & \text { if } t_{i} \leq u \leq t_{i+1} \\ =0 & \text { elsewhere }\end{cases}
$$

To complete the Basis function the values of $k$ and $i$ need to be defined where $k$ is the order of the curve. For continuity purposes $k$ is taken as 3 or 4 since a B-spline's continuity is defined by the equation (55):

$$
C^{k-2}
$$

As for $i$ it takes the range from 0 to $n$, where $n=p-1$ and $p$ is the number of control points defined on the path, so the overall B-spline function is simplified and shown below in equation (56):

$$
C(u)=\sum_{i=0}^{n} N_{i, k}(u) P_{i}
$$

The curves are used to replace circles in the previously designed road and their influence on handling behaviour is compared, by utilising the cost function.

\section{DISCUSSION}

The results of the simulation in terms of Handling behaviour are summarised in Table IV where a smaller value indicates more comfortable handling. The results indicate that all transition curves produce better handling comfort as compared to the "worst path".

TABLE IV

HANDLING COMFORT VALUE USING THE COST FUNCTION

\begin{tabular}{|c|ccc|}
\hline & Hermite & Bezier & B-spline \\
\hline $3 \mathrm{pt}$ & 10.924 & 10.926 & 10.922 \\
\hline Controller used & MPC & MPC & MPC \\
\hline $4 \mathrm{pt}$ & N/A & 11.43 & 11.40 \\
\hline Controller used & N/A & SMC & MPC \\
\hline $5 \mathrm{pt}$ & N/A & 13.33 & 19.70 \\
\hline Controller used & N/A & MPC & SMC \\
\hline
\end{tabular}

In terms of points defined per curve, it is evident that defining a curve with less number of points produces better handling as 3-point curves produces the lowest values in the cost function regardless of the transition curves being used. In terms of controllers, curvature independent controller (MPC) produces better handling than curvature dependent controller (SMC). MPC controller produced 5 out 7 top scenarios, including the lowest handling value. Another interesting fact noticed, was that curvature independent controller produces identical values for curves defined by 3 -points indicating that curvature independent controllers suppress distinct features of the curves.

More importantly the best transition curve that produces the best handling overall is difficult to choose from our cost function when it comes to 3-point curves (as all produce very similar values) but as we move onto 4-point curves it seems that the B-spline curve performs slightly better than the rest. However, at 5-point defined curves Bezier out perform Bspline curves quite handsomely. It should be noted that authors do not recommend path planning of curves with 5-points for two reasons; 1) Higher handling values are produced by these curves, 2)They are not computationally feasible as a result of high orders of the curves. Hence, the usage of B-spline curves with 3 or 4-points in tandem with curvature independent controllers like MPC are recommended for better handling performance and computational efficiency.

\section{CONCLUSION}

In summary the present study addresses the issue of handling behaviour of an autonomous vehicle by defining a cost function built on 3 objective functions, namely: FFT values of steering angles, VDV of lateral and roll accelerations and lateral path tracking error. These values were later calculated by designing a reference track and routing it with different transition curves through dependent and independent controllers. Results indicate that all transition curves produce more comfortable handling as compared to the "worst path'. As for 
3-point curves the handling behaviour of all curves is the same due to the nature of the controller being used, but for 4-point defined curves it is seen that B-spline curves produce the best handling behaviour.

Results serve as evidence indicating B-splines are the most capable transition curve for producing comfortable handling behaviour in autonomous vehicles. Further investigating characteristics of B-splines like local control and non-uniformity (NURBS- Non uniform rational Basis Spline) might further improve the comfort in autonomous vehicles in terms of handling behaviour.

\section{REFERENCES}

[1] S. O.-R. A. V. S. Committee et al., "Taxonomy and definitions for terms related to on-road motor vehicle automated driving systems," $S A E$ Standard J, vol. 3016, pp. 1-16, 2014.

[2] T. E. Trimble, R. Bishop, J. F. Morgan, M. Blanco, et al., "Human factors evaluation of level 2 and level 3 automated driving concepts: Past research, state of automation technology, and emerging system concepts," 2014

[3] J. Weyer, R. D. Fink, and F. Adelt, "Human-machine cooperation in smart cars. an empirical investigation of the loss-of-control thesis," Safety science, vol. 72, pp. 199-208, 2015.

[4] M. Elbanhawi, M. Simic, and R. Jazar, "In the passenger seat: investigating ride comfort measures in autonomous cars," IEEE Intelligent Transportation Systems Magazine, vol. 7, no. 3, pp. 4-17, 2015.

[5] C. G. L. Bianco, "Minimum-jerk velocity planning for mobile robot applications," IEEE Transactions on Robotics, vol. 29, no. 5, pp. 13171326, 2013.

[6] Y. Morales, N. Kallakuri, K. Shinozawa, T. Miyashita, and N. Hagita, "Human-comfortable navigation for an autonomous robotic wheelchair," in 2013 IEEE/RSJ International Conference on Intelligent Robots and Systems. IEEE, 2013, pp. 2737-2743.

[7] N. Mohajer, S. Nahavandi, H. Abdi, and Z. Najdovski, "Enhancing passenger comfort in autonomous vehicles through vehicle handling analysis and optimization," IEEE Intelligent Transportation Systems Magazine, 2020.

[8] R. N. Jazar, "Mathematical theory of autodriver for autonomous vehicles," Journal of Vibration and Control, vol. 16, no. 2, pp. 253-279, 2010.

[9] H. Marzbani, A. Mohd Harithuddin, M. Simic, M. Fard, and G. Nakhaie Jazar, "Steady-state dynamic steering," in Smart Digital Futures 2014 (SDF-14). IOS Press, 2014, pp. 493-504.

[10] H. Marzbani, H. Khayyam, C. N. TO, V. Quoc, and R. N. Jazar, "Autonomous vehicles: Autodriver algorithm and vehicle dynamics," IEEE Transactions on Vehicular Technology, vol. 68, no. 4, pp. 3201$3211,2019$.

[11] H. Marzbani, R. N. Jazar, and M. Fard, "Steady-state vehicle dynamics," in Nonlinear approaches in engineering applications. Springer, 2015, pp. 3-30.

[12] S. Milani, H. Marzbani, A. Khazaei, and R. N. Jazar, "Vehicles are lazy: On predicting vehicle transient dynamics by steady-state responses," in Nonlinear Approaches in Engineering Applications. Springer, 2020, pp. 3-38.

[13] J.-J. E. Slotine, W. Li, et al., Applied nonlinear control. Prentice hall Englewood Cliffs, NJ, 1991, vol. 199, no. 1.

[14] R. N. Jazar, Vehicle Planar Dynamics. Cham: Springer International Publishing, 2017, pp. 565-670. [Online]. Available: https://doi.org/10. 1007/978-3-319-53441-1_9

[15] S. Tajeddin, "Mpsee (https://www.mathworks.com/matlabcentral/fileexchange/64608mpsee)," 2020.

[16] S. Milani, H. Marzbani, A. Khazaei, M. Simic, and R. N. Jazar, "Elliptical combined-slip tire model in vehicle dynamics," in Innovation in Medicine and Healthcare Systems, and Multimedia. Springer, 2019, pp. 457-468.

[17] K. Yang and S. Sukkarieh, "An analytical continuous-curvature pathsmoothing algorithm," IEEE Transactions on Robotics, vol. 26, no. 3, pp. $561-568,2010$.

[18] M. Elbanhawi, M. Simic, and R. Jazar, "Improved manoeuvring of autonomous passenger vehicles: Simulations and field results," Journal of Vibration and Control, vol. 23, no. 12, pp. 1954-1983, 2017.
[19] P. Mohan, GATE 2020 Civil Engineering Guide with 10 Practice Sets (6 in Book +4 Online) 7th edition. Disha publications, 2019.

[20] U.-Y. Huh and S.-R. Chang, "Ag 2 continuous path-smoothing algorithm using modified quadratic polynomial interpolation," International Journal of Advanced Robotic Systems, vol. 11, no. 2, p. 25, 2014.

[21] E. Magid, D. Keren, E. Rivlin, and I. Yavneh, "Spline-based robot navigation," in 2006 IEEE/RSJ International Conference on Intelligent Robots and Systems. IEEE, 2006, pp. 2296-2301.

[22] J. Connors and G. Elkaim, "Analysis of a spline based, obstacle avoiding path planning algorithm," in 2007 IEEE 65th Vehicular Technology Conference-VTC2007-Spring. IEEE, 2007, pp. 2565-2569.

[23] S. Gulati, C. Jhurani, B. Kuipers, and R. Longoria, "A framework for planning comfortable and customizable motion of an assistive mobile robot," in 2009 IEEE/RSJ International Conference on Intelligent Robots and Systems. IEEE, 2009, pp. 4253-4260.

[24] K. Jolly, R. S. Kumar, and R. Vijayakumar, "A bezier curve based path planning in a multi-agent robot soccer system without violating the acceleration limits," Robotics and Autonomous Systems, vol. 57, no. 1, pp. 23-33, 2009.

[25] B. Lau, C. Sprunk, and W. Burgard, "Kinodynamic motion planning for mobile robots using splines," in 2009 IEEE/RSJ International Conference on Intelligent Robots and Systems. IEEE, 2009, pp. 2427-2433.

[26] C. De Boor, "On calculating with b-splines," Journal of Approximation theory, vol. 6, no. 1, pp. 50-62, 1972. 